\title{
Comparison of Presumptive Blood Test Kits Including Hexagon OBTI
}

Emma Johnston, ${ }^{1}$ M.Sc.; Carole E Ames, ${ }^{2 *}$ Ph.D.; Kathryn E Dagnall, ${ }^{2}$ M.Sc.; John Foster, ${ }^{2}$ B.Sc.; and Barbara E Daniel, ${ }^{1}$ Ph.D.

${ }^{1}$ Department of Forensic Science and Drug Monitoring, King's College London, London, UK ${ }^{2}$ Directorate of Forensic Services, Metropolitan Police Service, London, UK

* Corresponding Author - Directorate of Forensic Services, Metropolitan Police Service, New Scotland Yard, 10 Broadway, London, SW1H OBG, UK 
ABSTRACT: Four presumptive blood tests, Hexagon OBTI, Hemastix, Leucomalachite green (LMG), and Kastle-Meyer (KM) were compared for their sensitivity in the identification of dried bloodstains. Stains of varying blood dilutions were subjected to each presumptive test and the results compared. The Hexagon OBTI buffer volume was also reduced to ascertain whether this increased the sensitivity of the kit. The study found that Hemastix was the most sensitive test for trace blood detection. Only with the reduced buffer volume was the Hexagon OBTI kit as sensitive as the LMG and KM tests. However, the Hexagon OBTI kit has the advantage of being a primate specific blood detection kit. This study also investigated whether the OBTI buffer within the kit could be utilized for DNA profiling after presumptive testing. The results show that DNA profiles can be obtained from the Hexagon OBTI kit buffer directly.

KEYWORDS: forensic science, presumptive blood testing, hexagon OBTI, kastle- meyer, leucomalachite green, hemastix ${ }^{\circledR}$ 
Presumptive blood tests are conducted at a scene of crime after visual examination and prior to submission to the laboratory for genetic analysis. They save time and money by prioritizing the samples sent for DNA analysis. Ideally, a test to detect body fluids should be human specific, sensitive and robust enough to be used at crime scenes.

Many presumptive tests for blood are color catalytic tests based upon the peroxidase-like activity of the hemoglobin $(\mathrm{Hb})$ moiety found within red blood cells (1). This type of test includes the Kastle-Meyer (KM) test, leucomalachite green (LMG) and Hemastix ${ }^{\circledR}$. However, new tests are now available based upon immunological methods. These include Heme Select ${ }^{\mathrm{TM}}$ (SmithKline Diagnostics, San Jose, CA), Hexagon OBTI (Gesekkschaft fur Biochemica und Diagnostic mbH, Wiesbaden, Germany) and ABAcard $\AA$ HemaTrace ${ }^{\circledR}$ (Abacus Diagnostic, Brisbane, Australia). All these kits use anti- human $\mathrm{Hb}$ antibodies, to provide a means of detection for the presence of human (primate) $\mathrm{Hb}$. The advantage of these tests, compared with the color catalytic tests, is that they do not give false positive results due to the presence of other peroxidases and are primate specific; this can be important when dealing with crime in a domestic setting where blood other than human blood may be present.

In this study the Hexagon OBTI kit was tested against the currently used standard procedures within the Metropolitan Police Service. The specificity of the Hexagon OBTI test to primates has been validated by Hochmeister et al. (2). Other potential sources for cross-reactivity were tested and only ferret blood was shown to react (3). The Hexagon OBTI kit was designed for the detection of occult blood in fecal samples and works by adding the sample to 
a bottle containing $2 \mathrm{~mL}$ buffer. The buffer is then tested for the presence of hemoglobin by applying of two drops of buffer to the test cartridge containing free monoclonal anti- human $\mathrm{Hb}$ antibodies labeled with blue dye particles. On addition of a sample containing human $\mathrm{Hb}$, an immunocomplex is formed between the human $\mathrm{Hb}$ and the marked antibodies. This immunocomplex migrates along the test strip to an area where polyclonal anti- human $\mathrm{Hb}$ antibodies are immobilized. These bind to the previously formed immunocomplex creating an antibody-antigen-antibody sandwich. This in turn concentrates the dye and a blue line is formed.

Hochmeister et al. (2) first conducted a validation study of the Hexagon OBTI test for forensic use and their method was extended by the work of Hermon et al. (4).

In this study the Hexagon OBTI test was initially compared with three color catalytic tests (KM, LMG and Hemastix®) for sensitivity. The second part of the study focussed specifically on the Hexagon OBTI kit. This aimed to extend previous research by examining whether it would be possible to directly obtain a DNA profile from the buffer post blood identification. 


\section{Methods}

Comparison of Presumptive Blood Kits

Bloodstains were created on white tiles using varying volumes of fresh neat blood from one volunteer $(0.1 \mu \mathrm{L}, 1 \mu \mathrm{L}$ and $50 \mu \mathrm{L})$. Also, $50 \mu \mathrm{L}$ aliquots of blood diluted with sterile water to varying dilutions (1 in 10, 1 in 50, 1 in 100, 1 in 250, 1 in $1 \times 10^{3}, 1$ in $5 \times 10^{3}, 1$ in $1 \times 10^{4}, 1$ in $5 \times 10^{4}$ and 1 in $1 \times 10^{5}$ ) were aliquoted in the same manner. All stains were created in triplicate and allowed to dry overnight. Each stain was tested using the four kits and the results recorded. Each kit was used according to the manufacturer's instructions. The KM test was conducted as follows: the stain was rubbed gently with filter paper. A drop of ethanol was added to the paper followed by 1- 2 drops of $\mathrm{KM}$ solution. After a brief interval, to ensure that no color developed, several drops of hydrogen peroxide were added. An immediate pink color was indicative of blood. The LMG test procedure is similar; the stain was rubbed gently with filter paper. LMG solution (2 drops) was added to the paper. After ensuring that no color developed, several drops of hydrogen peroxide were added and the green color change is indicative of blood. When using the Hemastix ${ }^{\circledR}$ kit, the strip was rubbed onto the stain and one drop of distilled water was added to the strip. After $60 \mathrm{sec}$, the color of the strip was compared with an indicator color chart. A change from yellow to green is indicative of blood. The Hexagon OBTI kit contains an applicator inside the lid of the buffer bottle. This was used to scrape the stain three times and then was placed back inside the buffer. The bottle was shaken for $10 \mathrm{sec}$ by hand. Two drops of buffer solution were applied to the test strip and the test result (blue line in test and control zone) was read after 10 min. 
To try and increase the sensitivity of the test for certain dilutions of blood (1 in $5 \times 10^{3} ; 1$ in $1 \times 10^{4} ; 1$ in $5 \times 10^{4} ; 1$ in $1 \times 10^{5}$ ) the volume of the buffer was reduced from $2 \mathrm{~mL}$ to $200 \mu \mathrm{l}$. Reduction of the buffer volume had been shown to increase sensitivity by Hermon et al. (4).

\section{DNA Profiling of Blood Samples Collected in Hexagon OBTI Kit Buffer}

This part of the study focussed specifically on the Hexagon OBTI kit. Preliminary tests established that the Hexagon OBTI buffer does not inhibit standard DNA profiling. Blood was added to the Hexagon OBTI buffer (2mL) to obtain various dilutions ( 1 in 10,1 in 100,1 in $1 \times 10^{3}$ and 1 in $1 \times 10^{4}$ ). Aliquots $(200 \mu \mathrm{L})$ of each blood/buffer dilution were subjected to DNA extraction using the QIAamp DNA mini kit (Qiagen, Hilden, Germany) according to manufacturer's instructions. DNA was eluted from the mini kit column with $200 \mu \mathrm{L}$ of $\mathrm{ddH}_{2} \mathrm{O}$ and $5-10 \mu \mathrm{L}$ (depending on concentration) was amplified using AmpFISTR® SGMplus kit (Applied Biosystems, Foster City, CA ) in a $25 \mu \mathrm{L}$ PCR reaction on the PE GeneAmp 9700 (Applied Biosystems). This was followed by DNA profiling of $1.5 \mu \mathrm{L}$ amplicons using the $\mathrm{ABI} 310$ Genetic Analyser (Applied Biosystems).

Higher blood dilutions were also included to try and assess the highest dilution of blood and OBTI buffer that DNA can be profiled from. Blood dilutions of 1 in $1 \times 10^{5}, 1$ in $1 \times 10^{6}$ and 1 in $1 \times 10^{7}$ were first concentrated using YM-100 Centricon ${ }^{\circledR}$ units (Millipore, Billerica, MA) from $2 \mathrm{~mL}$ to $c$. $600 \mu \mathrm{L}$. The DNA from this was then extracted using the QIAamp DNA mini kit (Qiagen) and eluted in $200 \mu \mathrm{L}$ of $\mathrm{ddH}_{2} \mathrm{O}$ according to manufacturer's protocols. The DNA extract was then concentrated using YM-100 Microcon $®$ units 
(Millipore) to c. $20 \mu \mathrm{L}$ before amplification and profiling as for the other dilutions.

Blood/buffer samples were stored at $-20^{\circ} \mathrm{C}$ for $2-5$ days until use, but to assess the longevity of samples, three samples of 1 in 10, 1 in 100, 1 in $1 \mathrm{x}$ $10^{3}$ and 1 in $1 \times 10^{4}$ blood/buffer dilutions were kept at $\sim 4^{\circ} \mathrm{C}$ for 2 months and then subjected to DNA extraction, amplification and profiling as for the equivalent dilutions above.

\section{Results and Discussion}

Comparison of Presumptive Blood Kits

The results of the diluted bloodstains are presented in Table 1. These results demonstrate that Hemastix ${ }^{\circledR}$ was the most sensitive of the four kits examined in this study. It was able to detect a $50 \mu \mathrm{L}$ aliquot of blood at a 1 in $5 \times 10^{4}$ dilution. KM and LMG both had a limit of detection of 1 in $1 \times 10^{4}$. Using the Hexagon OBTI kit according to manufacturer's instructions (no reduction of buffer volume) gave a limit of detection of 1 in $1 \times 10^{3}$. For Hexagon OBTI to have equal sensitivity to $\mathrm{KM}$ and LMG, the buffer volume in the collection bottle had to be reduced from $2 \mathrm{~mL}$ to $200 \mu \mathrm{L}$. This has implications for crime scene examiners as they would have to prepare the kit before use. Even with this reduction in volume, this study found the kit not to be as sensitive as Hemastix ${ }$.

Hochmeister et al. (2) found the lower limit of detection for Hexagon OBTI to be 1 in $1 \times 10^{6}$ in the buffer when the incubation time was extended to $2 \mathrm{~h}$. In a later study, Hermon et al. (4) decreased the incubation time to 30 minutes 
and found the lower limit of detection to be 1 in 1000, comparable to the limit found in the current study. In this present study, the sample was immediately introduced to the test strip from the buffer thereby eliminating the incubation time altogether, as limited incubation is consistent with the conditions a scene of crime examiner would work under. It should be noted that this study used a nonporous substrate for test bloodstains. The sensitivities of each of the four tests from this work cannot necessarily be extended to porous substrates. Other researchers have used porous substrates. Hermon et al. (4) used gauze and Hochmeister et al. (2) used cotton cloth. As mentioned previously, the sensitivity of Hexagon OBTI found in this study and Hermon et al. (4) are of similar magnitude. Further work on the sensitivities of all four tests of blood on a variety of substrates should be conducted for a complete comparison of presumptive blood test sensitivities.

The Hexagon OBTI kit also gave false-negative results for neat blood and at 1 in 10 dilution; this is thought to be due to the high dose Hook effect as documented by Fernando and Wilson (5). The high dose hook effect occurs when there is an excess of antigen compared to antibody. The effect causes a paradoxical decrease in response and the phenomenon is named after the hook that is observed in the dose response curve when data are plotted as a signal versus antigen concentration. The high dose hook effect does not occur with the three color catalytic tests utilized in this study.

However, the advantages of Hexagon OBTI and other immunological kits should not be dismissed. Namely, the specificity makes this type of kit preferential in situations where non-primate blood may also be present. Also, 
the lack of reactivity with other oxidants (such as bleach) means that the Hexagon OBTI kit does not produce false positives in situations where the three color catalytic tests used in this study may do.

\section{DNA Profiling of Blood Samples collected in Hexagon OBTI Buffer}

This preliminary study has demonstrated that the blood remaining in the Hexagon OBTI collection bottle can be used in subsequent DNA profiling, thus providing a direct link to the crime scene stain. The quality of DNA profile obtained for each of the blood dilutions is presented in Table 2 . Profiles deemed suitable for inclusion on the UK National DNA database (NDNAD) were obtained from all samples of the 1 in 10 dilution through to the to 1 in $1 \times 10^{5}$ dilution. It should be noted that this dilution is actually lower than the lower detection limit of the blood test even after reducing the collection buffer to $200 \mu \mathrm{L}$ (Table 1 ). The less concentrated blood samples either produced poor partial profiles or no profiles, despite the methodology for these samples including concentration steps. Whilst this study indicates that DNA profiles can be obtained from blood in the Hexagon OBTI buffer solution, this study did not consider whether the buffer solution reduces the amount of DNA recovered and this should be considered in future work.

DNA profiles could be obtained from blood/buffer samples stored for 2 months at $4^{\circ} \mathrm{C}$. When compared to equivalent blood dilutions that were stored at $4^{\circ} \mathrm{C}$ for up to 5 days the total profile peak area was found to be significantly less. These preliminary results suggest that if profiling cannot be conducted immediately that the blood/buffer solution should be stored at $-20^{\circ} \mathrm{C}$ until use. 
This study did not include examination of the longevity of samples at $-20^{\circ} \mathrm{C}$ and this should be included in further longevity studies.

\section{Conclusion}

Due to the lower sensitivity when compared with three color catalytic tests, the Hexagon OBTI kit is not recommended for searching for blood at crime scenes. However, the kit could be used in conjunction with the other tests. By utilizing the kit after locating blood with a color catalytic test, it would reaffirm the positive result and would ascertain whether located stains are human blood. Also the buffer could be used for DNA profiling as demonstrated in this study.

\section{Acknowledgements}

The authors thank the staff at Forensic Science Service (Lambeth) for providing LMG solution. The authors would also like to thank the volunteer who donated blood. This work was completed by EJ as apart of her MSc Forensic Science (King's College, London) project placement with the Metropolitan Police Service. 


\section{References}

1. Lee HC, Pagliaro EM. Serology: blood identification. In: Siegel J, Knupfer

G, Saukko P, editors. Encyclopaedia of forensic sciences. San Diego: Academic Press, 2000;1331-8.

2. Hochmeister MN, Budowle B, Sparkes R, Rudin O, Gehrig C, Thali M, et al. Validation studies of an immunochromatographic 1-step test for the forensic identification of human blood. J Forensic Sci 1999;44(3):597-602.

3. Rowley BO. Commentary on: Hochmeister MN, Budowle B, Sparkes R, Rudin O, Gehrig C, Thali M, Schmidt L, Cordier A, Dirnhofer R. Validation studies of an immunochromatographic 1-step test for the forensic identification of human blood. J Forensic Sci 1999;44(6):1323.

4. Hermon D, Shpitzen M, Oz C, Glattstein B, Azoury M, Gafny R. The use of the Hexagon OBTI test for detection of human blood at crime scenes and on items of evidence. Part 1: validation studies and implementation. J Forensic Ident 2003;53(5):566-75.

(5) Fernando SA and Wilson GS. Studies of the 'hook' effect in the one-step sandwich immunoassay. J Immunol Methods 1992;151:47-56. 
Additional Information - Reprints Not Available from Author

Carole Ames, Ph.D.

Directorate of Forensic Services

Metropolitan Police Service

New Scotland Yard

10 Broadway

London

SW1H OBG

U.K. 
TABLE 1 - Comparison of the sensitivity of four presumptive blood test kits on $50 \mu \mathrm{L}$ of dilute blood. * Result after reducing the volume of buffer in collection bottle from $2 \mathrm{~mL}$ to $200 \mu \mathrm{L} . \quad+=$ positive result $\quad$ - = negative result. 


\begin{tabular}{|c|c|c|c|c|}
\hline Blood Dilution & KM & LMG & Hemastix ${ }^{\circledR}$ & Hexagon OBTI \\
\hline Neat & + & + & + & - \\
\hline 1 in 10 & + & + & + & - \\
\hline 1 in 50 & + & + & + & + \\
\hline 1 in 100 & + & + & + & + \\
\hline 1 in 250 & + & + & + & + \\
\hline 1 in $1 \times 10^{3}$ & + & + & + & + \\
\hline 1 in $5 \times 10^{3}$ & + & + & + & $+^{\star}$ \\
\hline 1 in $1 \times 10^{4}$ & + & + & + & $+^{\star}$ \\
\hline 1 in $5 \times 10^{4}$ & - & - & + & - \\
\hline 1 in $1 \times 10^{5}$ & - & - & - & - \\
\hline
\end{tabular}


TABLE 2- DNA profiling results for varying dilutions of blood in the Hexagon OBTI collection buffer. For dilutions 1 in 10 to 1 in $1 \times 10^{4}, 200 \mathrm{~mL}$ of the buffer/blood mix was subjected to DNA extraction. The other dilutions were first concentrated and then the whole sample was subjected to DNA extraction. 


\begin{tabular}{|c|c|}
\hline Blood Dilution & Quality of DNA profile \\
\hline 1 in 10 & Full \\
\hline 1 in 100 & Full \\
\hline 1 in $1 \times 10^{3}$ & Full \\
\hline 1 in $1 \times 10^{4}$ & Full \\
\hline 1 in $1 \times 10^{5}$ & Loadable partial \\
\hline 1 in $1 \times 10^{6}$ & Poor partial \\
\hline 1 in $1 \times 10^{7}$ & No Profile \\
\hline 1 in $1 \times 10^{8}$ & No Profile \\
\hline 0 & No Profile \\
\hline
\end{tabular}

